\title{
Globe
}

Revue internationale d'études québécoises

\section{Michel Vaïs (dir.) : Dictionnaire des artistes du théâtre québécois, Montréal, Cahiers de théâtre Jeu/Québec Amérique, 2008}

\section{Rachel Killick}

Volume 12, numéro 1, 2009

URI : https://id.erudit.org/iderudit/1000780ar

DOI : https://doi.org/10.7202/1000780ar

Aller au sommaire du numéro

Éditeur(s)

Globe, Revue internationale d'études québécoises

ISSN

1481-5869 (imprimé)

1923-8231 (numérique)

Découvrir la revue

Citer ce compte rendu

Killick, R. (2009). Compte rendu de [Michel Vaïs (dir.) : Dictionnaire des artistes du théâtre québécois, Montréal, Cahiers de théâtre Jeu/Québec Amérique, 2008] Globe, 12(1), 190-193. https://doi.org/10.7202/1000780ar d'utilisation que vous pouvez consulter en ligne.

https://apropos.erudit.org/fr/usagers/politique-dutilisation/ 
Malgré quelques réserves sur certaines redondances de principes et d'axiomes et sur le caractère parfois discutable des lectures personnelles des romans, il faut reconnaittre que le livre de Martine-Emmanuelle Lapointe est une contribution riche et complexe à la critique du champ culturel et de l'institution littéraire au Québec. Il apporte surtout une réflexion sérieuse, sensible aux interrelations entre théorie et histoire littéraires, sur les postures critiques et les conflits d'interprétation qui ont balisé un demi-siècle de la réception au Québec de ces trois romans consacrés "classiques " dans les années 1960. Cherchant surtout à objectiver ce que les Québécois (savants et profanes) voulaient $y$ voir pour narrer sur eux-mêmes, Martine-Emmanuelle Lapointe ne renverse pas ce "récit mémoriel ", mais le considère comme un passage obligé vers ce que François Ricard a appelé la " normalisation d'une littérature ".

Józef Kwaterko Université de Varsovie

\section{Michel Vaïs (dir.) \\ Dictionnaire des artistes du théatre québécois Montréal, Cahiers de thêâtre Jeu/Québec Amérique, 2008.}

Ce beau volume consacré aux artistes de la scène comble une lacune de taille dans la documentation de l'activité théâtrale québécoise, limitée jusqu'ici à des répertoires, vieux de trente ou quarante ans, d'auteurs de theâtre ou de leurs textes, ou aux informations plus diverses, mais éparpillées et parfois peu fiables, disponibles de nos jours sur Internet. Michel Vaïs, éditeur des Cabiers de théatre Jeu, s'est fixé le but de développer les indications de l'affiche L'arbre du theátre québécois (2001) en un ouvrage de référence, dont la sortie, prévue pour 2006, devait souligner du même coup le $30^{\mathrm{e}}$ anniversaire du périodique qu'il dirige. Un manque de ressources en a retardé la publication et ce n'est que grâce à un appel vigoureux au parrainage, lancé par Vaïs dans une conférence de presse en 2006, et à la générosité de la réponse (des individus pour la plupart), que le dictionnaire a pu enfin voir le jour.

Toute entreprise de publication - et particulièrement celle d'un dictionnaire - doit composer avec les contraintes financières qui lui sont imposées. Comment en effet choisir dans le milieu tellement riche de l'activité théâtrale québécoise ? Vaïs, dans son introduction, évoque les 
multiples échanges avec les lecteurs de Jeu et avec toute la gamme des professionnels, critiques, et observateurs du théâtre québécois, consultation qui a conduit à une première liste d'entrées éventuelles (plus de 1000 noms, d'après un article glissé dans le dossier de presse qui accompagnait l'ouvrage $\left.{ }^{15}\right)$. À partir de cette liste, le comité de rédaction de Jeu s'est livré à un tri rigoureux gouverné par trois critères essentiels : n'inclure que des artistes et des artisans du thêâtre ; inclure tout artiste qui, œuvrant au Québec, a marqué de façon définitive le théâtre québécois, quel que soit son lieu de naissance ; se concentrer sur le $\mathrm{XX}^{\mathrm{e}}$ siècle du théâtre québécois, et n'inclure que des artistes ayant au moins dix ans d'expérience. Cela a donné pour les 450 entrées retenues: 279 interprètes; 104 " metteurs en scène, pédagogues, directeurs artistiques, marionnettistes..."; 67 scénographes, dont 28 décorateurs; 16 " concepteurs de costumes, perruques, maquillages... "; 11 concepteurs d'éclairage et 12 concepteurs de sonorisation.

L'entrée pour chaque artiste est remarquable par la richesse des informations, comprenant, selon un protocole éditorial rigoureux : des précisions biographiques (date de naissance et, s'il y lieu, de décès); les détails essentiels de la formation au théâtre ; le nom des pièces dans lesquelles l'artiste a été impliqué (avec date, nom du thêâtre et/ou du metteur en scène) ; d'autres détails pertinents de son parcours (par exemple, participation aux productions de la télévision ou du cinéma, honneurs reçus); une brève appréciation de l'importance de son apport au théâtre québécois. Si on y retrouve tous les noms attendus (parmi les interprètes, Ovila Légaré, Gratien Gélinas, Jean Duceppe, Huguette Oligny, Denise Filiatrault, Monique Mercure ; parmi les metteurs en scène, André Brassard, Lorraine Pintal, Martine Beaulne; parmi les scénographes François Barbeau, Michel Beaulieu, Claude Goyette, pour n'en citer, au hasard, que quelques-uns), il y a aussi des noms peut-être moins généralement connus (certains interprètes de la première heure ou certains artistes de la coulisse), et parfois de nettes surprises, comme par exemple l'entrée traitant de la féministe Idola SaintJean, connue surtout pour son rôle dans les campagnes suffragistes des années 1920 et 1930 , mais présente ici en raison de ses activités de comédienne et de pédagogue.

Très intéressante chacune en elle-même, ces entrées individuelles permettent aussi, prises dans leur ensemble, de saisir les grandes lignes de l'évolution des réseaux de théâtre nationaux et internationaux au sein

$$
+4
$$

15. Matt RADZ, "A Hardcover Hall of Fame ", The Gazette, 21 janvier 2006, p. I2. 
desquels le theâtre québécois a développé un profil indépendant et original. Ainsi, au début, formation sur le tas mais aussi référence à la France avec, par exemple, le voyage exploratoire à Paris en 1925 de Fred Barry et d'Albert Duquesne, et, en 1937, la visite en France de leur troupe, première tournée en métropole d'une troupe canadienne, ou la formation à Paris du décorateur Claude Fortin ou de l'interprète Yvette Brind'Amour, future cofondatrice du Theâtre du Rideau Vert. Par la suite, généralisation de la formation professionnelle - à l'École nationale du thêatre du Canada, aux Conservatoires d'art dramatique de Montréal et de Québec, à l'université, ou dans les options théâtre des cégeps. Et, de pair avec cette évolution, et à partir surtout des années 1960, une fierté marquée pour les œuvres thêâtrales québécoises s'accompagnant d'une forte ouverture créatrice envers d'autres thêâtres et d'autres pensées théâtrales, comprenant, au-delà du thêâtre français toujours présent, la gamme des thêâtres autochtones, anglophones, européens, et asiatiques; sans oublier, autre richesse du thêatre québécois, l'apport important, tout au long de son histoire, de praticiens nés hors Québec, non seulement en France ou en pays francophone (la Belgique ou la Suisse), mais un peu partout ailleurs, de l'Angleterre à l'Argentine, de la Hongrie au Liban, de l'Italie à Israël, du Portugal à l'Ukraine.

Comme le souligne Vaiis au départ, un tel dictionnaire ne peut rien avoir d'exhaustif et les choix s'avèrent difficiles et délicats. Se trouvent ainsi inclus Maurice Podbrey, interprète anglophone, originaire de l'Afrique du Sud, présent en raison de sa contribution artistique au théâtre québécois comme cofondateur et directeur artistique du Centaur, mais exclue, Mercedes Palomino, dont l'activité au Théâtre du Rideau Vert est jugée moins artistique qu'organisatrice. Elle est présente cependant grâce à l'« Index des artistes cités n'ayant pas d'entrée dans cet ouvrage » (p. 413-19), comprenant 415 noms additionnels (artistes, artisans, auteurs, producteurs, critiques, gens du cinéma), la plupart Québécois, mais certains, des noms bien connus de personnalités internationales (tels Chaplin, Dullin, Marilyn Monroe, ou, nom plus récent, Liv Ullman ${ }^{16}$ ).

Le dictionnaire, très facile et très agréable à consulter, constitue ainsi un outil de recherche et de référence majeur, indispensable pour les

$+4+$

16. Un grand absent de cette liste : Michel Tremblay, dont le nom revient pourtant dans bien des entrées individuelles, mais dont les mentions, par mégarde, semblent avoir été assimilées à celle de son homonyme, Larry. Ainsi, puisque les auteurs de textes dramatiques, recensés ailleurs dans des dictionnaires de littérature québécoise, sont exclus du dictionnaire de Vaïs, Michel Tremblay n'a aucune entrée dans celui-ci. 
chercheurs, les professeurs et les étudiants, et d'un grand intérêt pour tout amateur du théâtre québécois. Il offre, présentées de façon claire et concise par une équipe de grands spécialistes en théâtre québécois, d'impeccables informations sur les artistes. En plus, dans son ensemble, il propose un portrait fascinant, fait de multiples " arrêts sur image ", du paysage dynamique de la représentation théâtrale québécoise au $\mathrm{XX}^{e}$ siècle. De très belles photos, aidant à la pleine appréciation du travail des artistes, s'ajoutent à la présentation très soignée du volume pour le plus grand plaisir du lecteur.

Rachel Killick Université de Leeds

\section{Pierre Nepveu \\ La poésie immédiate. \\ Lectures critiques 1985-2005 \\ Québec, Nota bene, 2008.}

"L'âme" : ce qui, en nous, têve toujours à la vie immédiate ${ }^{17}$ ". Telle était, en 1979, la conclusion du chapitre intitulé "L'évidence de la poésie ", l'une des toutes premières lectures critiques de Pierre Nepveu consacrée à Paul-Marie Lapointe. De la vie immédiate à la poésie immédiate, il n'y a qu'un pas, que franchit le poète et critique dans le nouveau livre qu'il fait paraître chez Nota bene. Ainsi décrit-il aujourd'hui la puissance de l'expérience de la poésie : "l'immédiateté poétique, là où le langage veut être la vie elle-même, dans sa crudité originelle, dans son déferlement insensé, déraisonnable " (p. 15). Il y a, dans cette qualité d'immédiateté, un précipité de présence qui attire Nepveu. La poésie immédiate, c'est celle qui décontenance, accule au mur du sens, marque et creuse le corps ; c'est aussi celle qui s'approche de la vie brute, de la vie sans filtre, de la vie sans les mots paradoxalement. En référence à Éluard, le titre de l'ouvrage indique donc l'événement absolu et le choc constamment renouvelé que demeure, même pour les plus chevronnés des exégètes, la lecture de poésie.

Au cours des trente dernières années, Pierre Nepveu n'aura eu de cesse de s'exposer au "vent charnel " et à la " pure violence " de la poésie (p. 15). Après une série de recensions dans Livres et auteurs québécois, de

$$
+*+
$$

17. Pierre NePveU, Les mots à l'écoute. Potsie et silence chez Fernand Ouellette, Gaston Miron et Paul-Marie Lapointe, Québec, Nota bene, 2002, p. 340. 